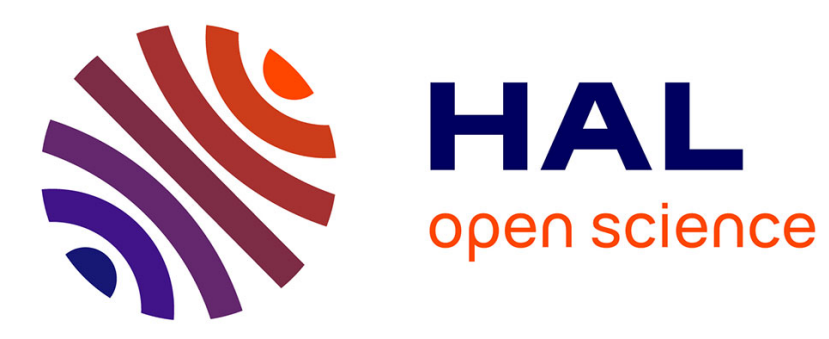

\title{
Optimal Probabilistic Energy-Aware Routing for Duty-Cycled Wireless Sensor Networks
}

Evangelia Tsiontsiou, Bernardetta Addis, Ye-Qiong Song, Alberto Ceselli

\section{To cite this version:}

Evangelia Tsiontsiou, Bernardetta Addis, Ye-Qiong Song, Alberto Ceselli. Optimal Probabilistic Energy-Aware Routing for Duty-Cycled Wireless Sensor Networks. 8th IFIP International Conference on New Technologies, Mobility and Security (NTMS 2016), Nov 2016, Larnaca, Cyprus. hal-01404169

\section{HAL Id: hal-01404169 \\ https://hal.inria.fr/hal-01404169}

Submitted on 28 Nov 2016

HAL is a multi-disciplinary open access archive for the deposit and dissemination of scientific research documents, whether they are published or not. The documents may come from teaching and research institutions in France or abroad, or from public or private research centers.
L'archive ouverte pluridisciplinaire HAL, est destinée au dépôt et à la diffusion de documents scientifiques de niveau recherche, publiés ou non, émanant des établissements d'enseignement et de recherche français ou étrangers, des laboratoires publics ou privés. 


\section{Optimal Probabilistic Energy-Aware Routing for Duty-Cycled Wireless Sensor Networks}

\author{
Evangelia Tsiontsiou, Bernardetta Addis, Ye-Qiong Song \\ LORIA - Université de Lorraine \\ Campus Scientifique, B.P. 239, 54506, Vandoeuvre-lès-Nancy, FRANCE \\ Emails: \{evangelia.tsiontsiou@loria.fr, bernardetta.addis@loria.fr, \\ ye-qiong.song@loria.fr\}
}

\author{
Alberto Ceselli \\ Università degli Studi di Milano \\ Dipartimento di Informatica \\ Via Bramante 65, 26013, Crema, ITALY \\ Email: \{alberto.ceselli@unimi.it\}
}

\begin{abstract}
Network lifetime is still the key issue when we deploy wireless sensor networks and IoT solutions in realworld applications. Current WSN research trends include dutycycling at MAC layer and energy efficient routing at network layer, among others. In this study we propose an Optimal Probabilistic Energy-Aware Routing Protocol (OPEAR) for dutycycled WSNs which aims at maximizing the network lifetime by keeping low energy consumption and balancing network traffic between nodes. Our experimental campaign reveals that our OPEAR protocol outperforms the popular Energy Aware Routing Protocol (EAR) from the literature, proving to be more effective in extending the network lifetime.
\end{abstract}

\section{INTRODUCTION}

Wireless Sensor Networks (WSNs) are pervasive. Diverse domains such as environmental control in buildings, healthcare, internet of things, robot control in automatic manufacturing environments exploit WSNs as a key component. A common issue in all WSNs applications is network lifetime: sensors have often limited battery, and recharging operations, if at all possible, substantially increase the cost of service.

While out-of-battery sensor failures, and therefore possible disconnections of portions of the network, may represent a simple loss in quality of service in some context, they may yield critical conditions in others. It is the case of WSNs for smart homes: these are an emerging technology for home health care assistance, that provides ambient assisted living services for elderly. Figure 1 a shows a typical scenario of a smart home equipped with different wireless sensor nodes, eventually enriched by an assistant robot, for permanently monitoring and assisting the person's daily life activities and personal health state evolution [17]. Data are collected to a sink either periodically (e.g. temperature, humidity, luminosity) or following a specific event (e.g. door opening/closing, chair and bed pressure or movement detection). The data transmission reliability is enhanced thanks to a multi-hop mesh topology.

When deploying a WSN in such a scenario, one of the most important requirements is to be able to estimate the network lifetime, assuming to have an estimate of the data transmission demands, as the frequency of periodic data sources or the average packet generating rate of the event-triggered sensor sources. Many issues intervene in the design of good policies to manage these WSNs, such as the ability to extend the expected lifetime of the full network, to take into account worst-case situations and identify the most critical sensors, to efficiently use batteries, to balance sensor usage and so on.

In our previous implementation of such a smart apartment lab [17], battery level indicator is embedded in the data packet and is one of the services provided by our gateway called MPIGate. However, for any given traffic demand pattern, finding the maximal network lifetime is still an open issue since it depends on the used routing protocol and the underlying MAC protocol duty cycle, among others.

In this paper we focus on the topic of designing an energy-aware routing protocol for maximizing the network lifetime, while still fulfilling the data collection requirements. We propose an Optimal Probabilistic Energy Aware Routing (OPEAR) protocol that combines a mathematical model to produce optimized routing tables in an offline computation phase and a simple, yet energy efficient, routing policy during realtime operations. We implemented a prototype of the OPEAR protocol in the Contiki emulation environment above ContikiMAC duty-cycled protocol, using a stress-test computational campaign. We compared OPEAR with the popular Energy-Aware Routing protocol (EAR) from the literature [5], also implemented (by us) over ContikiMAC. The computational tests reveals that OPEAR clearly outperforms EAR, yielding substantially higher network lifetimes. It is worth to notice that the original paper of EAR only evaluated performances by assuming an ideal MAC protocol, without collisions, neither duty-cycle.

The main contributions of this paper are:

1) We consider a concrete duty-cycle MAC protocol (contikiMAC) in our optimization model, while the existing works only focus on energy balancing at routing level, without consider the duty-cycle, which however allows to save the main part of energy.

2) To the best of our knowledge, this paper is, among the existing probabilistic routing algorithms, the first work which provides optimal probabilities.

3) The proposed work is tested in an emulation tool, Contiki, using the real code that is executed on the sensors, having more accurate results than a simple simulation. 
In the following we first review the literature on energyaware routing (Section $\mathrm{II}$ ); then we outline the OPEAR protocol and we detail the mathematical models it relies on for optimal probability evaluation (Section III). Finally, we introduce a realistic stress-test dataset and we describe our experiments, simulations and results (Section IV). Conclusions and perspectives are briefly presented in Section $\mathrm{V}$.

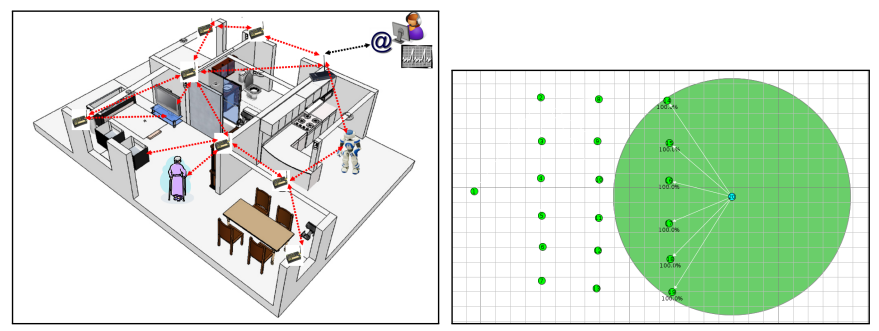

Fig. 1: a) A smart home scenario, b) Benchmark- 20-node graph.

\section{RELATED WORK}

Since nodes in WSNs are battery-powered, energy conservation is important to extend the functional lifetime of both individual nodes and the network. Several papers deal with the problem of finding the bounds of lifetime of a network. In [9], a flow optimization model is proposed for finding out the maximum lifetime of aggregating networks, assigning roles to the sensors. A similar approach is proposed in [10] where the authors maximize the lifetime imposing hop count constraints. Nevertheless, the above papers do not specify the way that their optimal solutions could be used in a routing process.

On the other hand, many routing protocols for WSNs exist in the literature that aim exclusively at preserving high levels of reliability and quality of service. The most common metric in probabilistic routing is the Expected Transmission Count (ETX), which is associated to the minimum hop count, and reliability parameters like Received Signal Strength Indicator (RSSI) and Link Quality Indicator (LQI). In [2], the authors use ETX in Destination-Sequenced Distance-Vector (DSDV) and Dynamic Source Routing (DSR) protocols. Also ExOR [3] uses ETX for assigning priorities in a set of candidate forwarders. In [4], a probabilistic routing scheme is proposed using a physical distance model for probability assignment.

While all the above protocols are reliability oriented, preserving energy is also addressed as an issue in the literature. In [11], a stochastic scheme is devised which randomly chooses next hops between those which are in the forwarding table. This approach turns out to be inefficient, neglecting quality checks in the selection of forwarders. In [6], the authors proposed an energy-efficient routing protocol based on AODV protocol by considering the transmission power and remaining energy capacity of the mobile nodes. The authors of [7] proposed probability based improved broadcasting algorithm which reduces the route request messages (RREQ) by using a broadcasting probability together with the consideration of the residual energy of nodes. In [8] the authors proposed the energy-efficient probabilistic routing (EEPR) algorithm, which employs both the ETX metric and the residual energy of each node as the routing metrics at the same time. In [5] an energyaware routing protocol (EAR) is introduced which chooses forwarders according to a neighbor cost. This neighbor cost includes the residual energy of the neighbor and also the cost of the whole possible path from the current node to the destination. This approach does not aim to find maximum energy paths but sub-optimal paths which leads to network lifetime increase.

Although the above papers seems to be energy efficient, in our paper we use different objectives for selecting forwarders. Our objective function maximizes the minimum residual energy and minimizes the maximum difference of residual energy between sensors at the same time so that the residual energy on sensors can be better balanced. EAR shares similar objectives with our proposal and for that reason it has been considered as a benchmark protocol for our performance comparison.

\section{OPEAR: AN OPTIMAL PROBABILISTIC ENERGY-AWARE ROUTING PROTOCOL}

The main idea of our OPEAR protocol is to maximize the lifetime of the network by (a) exploiting optimization models, and forecasting of transmission demands in terms of number of packets to be sent, to build optimized routing tables, and (b) limiting the realtime activity of sensors to basic operations, thereby limiting also their energy consumption.

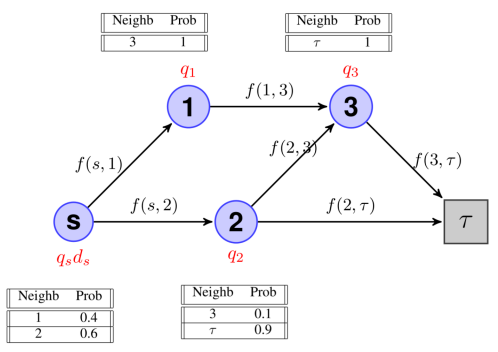

Fig. 2: An illustrating example.

As an overview, let us assume that our network is composed by three types of nodes: source nodes, representing sensors actually collecting data, relay nodes, representing sensors only acting as bridges, and a sink node, representing the terminal server, collecting all data. A sample network is depicted in Fig. 2, where $s$ is a source node, nodes 1,2,3 are relay nodes and $\tau$ represents the sink. An arc represent the possibility of a node to directly send packets to another one.

The OPEAR protocol can be summarized in three main phases.

1) Initialization Phase: The sink node having full knowledge of the network and an estimate of sensor transmission demands, calculates optimized routing tables. Then, it broadcasts a "hello" packet to the neighbors through flooding containing such information, so that each node can suitably fill its forwarding table. The "hello" packet 
is recursively transmitted from each sensor to its neighbors, eventually reaching the terminal sensors collecting data.

2) Data Forwarding Phase: Each source node collects and forwards data packets to a neighbor, that is randomly chosen according to the probabilities in its routing table. Each relay node receives packets and forwards them according to the same probabilistic policy. For increasing the reliability of the network, each node that receives a packet sends back an acknowledgment message. This procedure is iterated until the data packet reaches the sink node. Retransmissions in case of no-ACK is not taken into account in the optimization model, as we assumed that it is negligible in our setting. In case this is not true, the model can be extended to take into account such energy component. The energy consumption for the acknowledge message is taken into account.

3) Recalculation Phase: Optionally, either at fixed intervals or due to a triggering event, a recalculation phase is performed. As in the initialization phase the sink gathers information about the remaining energy of all the nodes and recomputes optimized routing tables. Then it broadcasts again hello packets for updating the sensor routing tables.

An example of the structure of routing tables is depicted in Fig. 2. The source node $s$ will send packets with probabilities 0.4 and 0.6 to nodes 1 and 2, respectively. The forwarding table of the node has the size of the number of neighbors of the node itself, so it is a quite compact information.

\section{A. Network Model and Problem Formalization}

In an offline planning phase probabilistic routing tables, one for each sensor, are computed by a centralized device. We assume that the centralized device has a global view on the network. In details, in such a planning phase we assume that:

- the network connecting sensors is static in terms of links (this is almost always the case for a small size home network where nodes are static and external interference is mitigated by the physical layer's channel hoping technique),

- a unique sink exists in the network (the sink is mainpowered without energy limitation), representing the destination of all packets (for higher reliability and more sensitive applications we can easily duplicate the sink),

- other sensor nodes are battery-powered with random initial energy budget,

- a planning module has full knowledge of the network (battery level of each sensor and radio links among sensor nodes, which can be obtained for instance using MPIGate gateway [17].),

- each sensor has local knowledge of the network, consisting in two sets of links, leading to forward and backward sensors, that is those sensors which are respectively nearer to and farther from the sink in terms of minimum number of hops to reach it (this can be the case of a

\begin{tabular}{c|c}
\hline Mode & Current $(\mathrm{mA})$ \\
\hline \hline TX & 17.4 \\
\hline RX & 18.8 \\
\hline Listen & 18.8 \\
\hline Sleep & 0.001 \\
\hline \hline
\end{tabular}

TABLE I: Radio current thresholds

DODAG as defined in IETF RPL protocol, where each node has its rank),

- an estimated demand is known, representing for each sensor the number of packets that must be routed in the whole planning period.

At the end of the offline planning phase the routing tables are transmitted to all sensors by means of a broadcasting operation. In particular, each sensor will receive a vector $p$, having one entry $p_{i}$ for each of its forward neighbors. In a real-time routing phase, instead, each sensor has only a local view, holding its own routing table and forwarding packets to its forwarder neighbors according to the probabilities specified therein.

\section{B. Energy Model}

The radio duty cycle is a critical component of the system regarding the power consumption. From the view of upper layer protocols, the sensors are usually assumed to have four states, i.e. transmitting, receiving, idle listening and sleeping and no cost for transitions between states [18]. Other researchers try to take more realistic factors into consideration [19] and others assume that since the nodes are expected to receive, transmit and listen at full power, for which current demands are approximately the same, two states of being in active or sleep mode can represent efficiently the radio operation [20].

For our experiments we used ContikiMAC radio duty cycle [16], therefore we take into account 4 states: transmitting, receiving, idle listening and sleeping. We assume that the nodes are synchronized through the phase-lock optimization mechanism. According to the CC2420 datasheet [21], we consider the current thresholds presented in Table If. The energy spent in each state is equal to that value multiplied to the duration of each state.The energy of overhearing (when a node is in the vicinity of a sender may receive and process the message in order to know if it is the destination or not) is a small share of the total consumed energy, and is therefore not taken into account in this work. Nevertheless, it can be easily added to the optimization model, and may therefore be subject of further investigation.

\section{Offline Optimization Model}

Let $G(N, A)$ be the graph representing the network, where the set of nodes $N$ represents the sensors, and the set of $\operatorname{arcs} A$ represents the wireless connections between the sensor nodes: the arc $(i, j)$ exists if sensor $i$ can communicate with sensor $j$. We denote by $\tau$ the sink node, collecting sensor data of all 
the network and by $N_{s} \subset N$ the set of source nodes, that are the sensors that collect data to be sent to the sink node. A source node can also receive and forward packets from other source nodes. We denote by $d_{i}$ the expected amount of data collected by sensor $i \in N_{s}$ within the planning horizon (total demand) and $q_{i}$ the energy the sensor $i \in N \backslash\{\tau\}$ at the beginning of planning. Note that, following assumptions III-A and the previous definitions, parameter $d_{i}$ is not defined for nodes that are not sources, and node energy is not defined for the sink node. The example in Fig. 2, represents the notation used in the graph representation and the following model. In red are reported the initial energy and (for sources only) the demand of each node. The problem of routing all data packets from nodes in $N_{s}$ to node $\tau$, maximizing the lifetime of the network, can be formulated as follows

$$
\begin{aligned}
& \text { maximize } \gamma(-z)+(1-\gamma) v \\
& \sum_{\substack{j \in N: \\
(i, j) \in A}} f_{i, j}^{k}=\sum_{\substack{j \in N: \\
(j, i) \in A}} f_{j, i}^{k} \quad \forall k \in N_{s}, \forall i \in N \backslash\{\tau, k\} \\
& \sum_{\substack{j \in N: \\
(i, j) \in A}} f_{i, j}^{i}=d_{i} \\
& \forall i \in N_{s} \\
& \sum_{\substack{i \in N: \\
(i, \tau) \in A}} \sum_{k \in N_{s}} f_{i, \tau}^{k}=\sum_{k \in N_{s}} d_{k} \\
& r_{i}=q_{i}-\sum_{k \in N_{s}}\left(\sum_{\substack{j \in N: \\
(i, j) \in A}} \varrho_{1} f_{i, j}^{k}+\right. \\
& \left.\sum_{\substack{j \in N: \\
(j, i) \in A}} \varrho_{2} f_{j, i}^{k}+\varrho_{3} T\right) \\
& \forall i \in N \backslash\{\tau\} \\
& r_{i} \leq q_{i} \\
& \forall i \in N \\
& v \leq r_{i} \\
& \forall i \in N \\
& r_{i}-r_{j} \leq z \\
& f_{i, j}^{k} \geq 0 \\
& r_{i} \geq 0
\end{aligned}
$$

where decision variables $f_{i, j}^{k}$ represent the number of packets whose source is $k$ to be sent from $i$ to $j$ (and symmetrically, the number of acknowledgment messages returned from $j$ to $i$ ), decision variables $r_{i}$ represent the residual energy (namely, initial energy minus consumed energy) at node $i$, variable $v$ represents the minimum residual energy and variable $z$ represents the maximum difference of residual energy between two sensors.

Constraints (1) impose flow conservation: each received packet must be forwarded. Constraints (2) and (3) impose respectively that all packets are forwarded from source sensors and all packets are received by the sink node. Constraints (4) link the energy consumption variable values to the number of packets sent and received; $\varrho_{1}$ represents the energy spent in each data packet transmission and $\varrho_{2}$ the energy spent in each data packet reception. The last term represents the fixed energy spent per second just because of duty-cycle (sleeping mode and CCA mechanism). Constraints (5) impose that not more than the available energy can be consumed. Constraints (6)-(7) ensure consistency between $r_{i}$ and $v$ and $r_{i}$ and $z$ variables, respectively. The energy which is supposed to be spent during sleep mode state (Table I) for all the planning period $T$ is removed in advance.

The objective function aims at simultaneously minimizing the maximum difference of residual energy between sensors and maximizing the minimum residual energy (to the aim of combining these two objectives, we need to reverse the first one, therefore we maximize $-z$ ). These two, potentially conflicting, objectives are balanced in a linear convex combination according to parameter $\gamma$. Indeed, in preliminary experiments we found out that considering only one objective at a time was leading to unbalanced networks at the end of the planning period.

Model (1) - 9) is compact, requiring an amount of memory which is polynomial in problem size. It can also be optimized in polynomial computing time, being either solved as a Linear Program [14], or by means of dedicated flow algorithms [15]. Furthermore, since data packets are identical for the flow structure and for the computation of probability tables, as discussed below, the commodity index on $f_{i, j}^{k}$ variables can be dropped, further reducing the computing effort needed to obtain optimal solutions

Let $f_{i, j}^{k, \star}$ be an optimal solution for the model above. We build a probabilistic routing table $P$ : each entry $P_{i j}$ is defined as follows

$$
P_{i j}= \begin{cases}\sum_{k \in N_{s}} \frac{f_{i, j}^{k, \star}}{\sum_{l:(i, l) \in A} \sum_{k \in N_{s}} f_{i, l}^{k, \star}} & \text { if }(i, j) \in A \\ 0 & \text { otherwise }\end{cases}
$$

The probability $P_{i j}$ corresponds to the fraction of demand forwarded to node $j$ by node $i$.

During broadcast, each sensor $i \in N$ stores as routing table a set of values $P_{i j}$ for all $j:(i, j) \in A$, representing the probability of forwarding a data packet to $j \in N$. We remark that no probabilistic routing table is required for backward reply with acknowledgment packets, as they are deterministically sent back to the forwarder. The same applies to the "hello" packets, that are deterministically broadcast from each sensor to all its backward neighbors.

\section{EXPERIMENTAL EVALUATION}

We performed an experimental campaign to assess the performances of our OPEAR protocol, comparing it with a benchmark protocol from the literature by means of tests on emulated networks. In the following we first detail the dataset we created, we describe our simulation setting and we finally present our results. The benchmark protocol can be further reviewed in [5].

\section{A. Dataset design}

The traffic patterns are generated periodically. As discussed in subsection III-A we assume that for a specific time period we can forecast the number of sensor packets that each sensor will send for this specific time period, that may depend on 
the type of sensor. In a practical setting for many sensor types, like periodic temperature monitoring, such an estimate is simple, while for others, like event-triggered movement sensors, application-dependent statistics must be considered.

We considered the grid-like topology depicted in Fig. 1] b. It corresponds to a high network density, in order to run tests in a worse-case scenario. We fixed the number of nodes to 20 , which is a common value for a smart-home application. Then we considered three cases: 1) only node 20 is a source, 2) nodes 20, 19 and 14 are sources, 3) all nodes are sources. Node 1 is set as sink and all the remaining nodes that are not sources are relay only.

As a stress test, we assumed to be in a scenario in which energy is scarce. To reproduce such a condition, we designed our test instances as follows. First, we generated random energy levels in the ranges [1000-2500] and [2000-3500] for single-source and multiple-source scenarios respectively, drawing values from uniform distributions. The energy ranges are chosen taking into account a desirable number of packets to be sent, to obtain significant simulations with a reasonable duration. Then we computed the maximum demand that can be routed without fully consuming the energy of any sensor. To perform such a computation we built the following model.

$$
\begin{array}{lc}
\sum_{\substack{j \in N: \\
(i, j) \in A}}^{\operatorname{maximize}} f_{i, j}^{k}=\sum_{\substack{j \in N: \\
(j, i) \in A}} f_{j, i}^{k} \quad \forall k \in N_{s}, \forall i \in N \backslash\{\tau, k\} & \forall i \in N_{s} \\
\sum_{\substack{j \in N: \\
(i, j) \in A}} f_{i, j}^{i}=\delta_{T} \phi_{i} & \\
\sum_{\substack{i \in N: \\
(i, \tau) \in A}} \sum_{k \in N_{s}} f_{i, \tau}^{k}=\delta_{T} \sum_{k \in N_{s}} \phi_{k} & \\
r_{i}=q_{i}-\sum_{k \in N_{s}}\left(\sum_{\substack{j \in N: \\
(i, j) \in A}} 2 \varrho_{1} f_{i, j}^{k}+\right. & \forall i \in N \backslash\{\tau\} \\
\left.\sum_{\substack{j \in N: \\
(j, i) \in A}} \varrho_{2} f_{j, i}^{k}+\varrho_{3} \delta_{T}\right) & \\
r_{i} \leq q_{i} \\
f_{i, j}^{k} \geq 0 \\
r_{i} \geq 0
\end{array} \quad \forall i \in N \backslash\{\tau\}
$$

Constraints (11), (12), (13), (14), (15), (16) and (17) have in turn the same meaning of constraints (1), (2), (3), (4), (5), (8) and (9), with the only important difference that $\delta_{T}$ is now a decision variable, that is maximized by our model.

Indeed that stress-test model shares features with that of [9]. However, objectives are different: in [9] the demands are known, and the network lifetime is maximized, while to build our instances we aim at finding the maximum amount of packets that the network can transmit before an out of battery failure occurs.

Finally, we created four instances, one for each of the following scenarios:

A random energy levels, single-source sending $D$ packets

\begin{tabular}{c|c}
\hline Settings & Value \\
\hline \hline Contiki version & 2.7 \\
\hline Wireless channel model & UDG Model with Distance Loss \\
\hline Communication range & $60 \mathrm{~m}$ \\
\hline Mote type & Tmote Sky \\
\hline Communication profile & Rime \\
\hline MAC Layer & CSMA \\
\hline Duty Cycle & ContikiMAC \\
\hline
\end{tabular}

TABLE II: Contiki OS and Cooja Parameters

B random energy levels, multiple homogeneous sources, each sending $D$ packets

C random energy levels, multiple heterogeneous sources, each sending a number of packets uniformly drawn in the range $[0.9 D, 1.1 D]$.

D random energy levels, all sources heterogeneous, each sending a number of packets uniformly drawn in the range $[0.9 D, 1.1 D]$.

\section{B. Emulation Scenario}

We emulated both protocols in Contiki 2.7 [22] using TelosB (also known as TMote Sky). This approach allows us to deploy in sensors the same computer code that would be executed in a real environment. Furthermore we used the COOJA simulator provided by Contiki [12], whose behavior is close to real hardware, thus yielding very reliable results together with the possibility of rapidly testing multiple scenarios. In Table III we report our COOJA settings.

\section{Results}

We first made a round of tests to assess the scalability of our OPEAR protocol. In particular, we measured the CPU time required to optimize the network flow models for creating the probabilistic routing tables as the size of the network increases. We verified that even problems on large networks can be optimized easily. This was expected, as only Linear Programming optimization is involved in the definition of the probabilistic routing tables. Then, as main performance measure we considered the network lifetime a protocol achieves, that is defined as follows.

Definition 4.1: The network lifetime is the time between the starting of sensor data transmission and the first sensor failure, considering a sensor to fail when its energy reaches zero.

Both protocols compute and assign probability tables during the initialization phase. During the main process, EAR calls the flooding process with a given frequency, while OPEAR can call the flooding process or not. In order to have a fair comparison between EAR and OPEAR, we considered two use settings: no flooding (nf), that is the probability tables are computed only once at the beginning of the simulation and never changed, and with flooding ( $\mathrm{kf}$, where $\mathrm{k}$ is the number of flooding steps performed), that is new probability tables are re-computed with a certain frequency, that is kept the same for both protocols. 
As far as parameters tuning is concerned, according to preliminary experiments we found EAR to perform better in our dataset with $\alpha=\beta=1.0$. The $\gamma$ parameter in OPEAR was set to 0.5 . Indeed, we could observe that with this setting, utopia values for both objective functions simultaneously could be attained; that was not the case by fixing either $\gamma=1.0$ or $\gamma=0.0$.

In table III the parameters for the different simulation experiments are summarized. Each column represent an emulation experiment and its use settings. In the second line the cases with different flooding cases are presented. A(nf), B(nf), C(nf) and $\mathrm{D}(\mathrm{nf})$ for case of no flooding, $\mathrm{A}(2 \mathrm{f}), \mathrm{B}(2 \mathrm{f}), \mathrm{C}(2 \mathrm{f})$ and $\mathrm{D}(2 \mathrm{f})$ for case of 2 flooding processes and $\mathrm{A}(4 \mathrm{f}), \mathrm{B}(4 \mathrm{f}), \mathrm{C}(4 \mathrm{f})$ and $\mathrm{D}(4 \mathrm{f})$ for case of 4 flooding processes.

In Figure 3 and 4 we report the network lifetime (vertical axis) obtained in each simulation (indicated in horizontal axis), expressed in msec. Blue bars correspond to the average results of the OPEAR protocol, while the red bars correspond to those of the EAR protocol. In each graph the first set of bars corresponds to the test without flooding (nf), the second corresponds to test with two flooding processes (2f), while the third set corresponds to test with four flooding processes (4f). Each experiment is repeated three times and we observed that there is no significant variation between the values of network lifetime among the tests. We also made experiments using two different random initial energies $q$ and $q^{\prime}$ to prove that this randomness does not affect the efficiency of our approach.

As a general consideration, we observe that OPEAR outperforms EAR in all simulations. OPEAR generates optimal probabilities, trying to minimize in part the difference of the residual energy of the nodes. This means that the nodes are used in that level so that at the end of the planning horizon they have the same residual energy. Therefore, our objective function prolongs the lifetime of the network. EAR chooses next hops by taking into account the residual energy of the next hop neighbor itself and the residual energy of nodes that may be part of the path till the packet arrives to the sink. In other words, EAR does not find paths with the most energy but sub-optimal paths which may include nodes with low energy. A drawback of EAR is that since the residual energy is normalized by the initial energy (that is a part of the cost function), the first round brings completely uniform probabilities among all the links, so nodes with lower energy are used at same level as nodes with higher energy.

Furthermore, flooding seems to have a positive influence in OPEAR, proving the capability of OPEAR of re-adapting routing tables to the changed panorama of sensor residual energy. Optimal solution let some sensors be used more than others with less energy. On the other hand, EAR does not seem to be improved significantly. We have to remark that the energy spent for flooding is not considered in our experiments. By that way, we are able to evaluate the mechanism of probability assignment to the links.

Table IV] present the variation of residual energy for both protocols. We can observe that OPEAR provides lower variation than EAR which proves the fact that OPEAR is able to keep more uniformly the residual energy of nodes.

OPEAR aims to balance the usage of sensors along a given planning period, thus improving lifetime. Our method is based on an approximation of ContikiMAC, predicting the energy that is only spent in CCA and transmitting-receiving phases by assuming ideal phase-lock mechanism provided by ContikiMAC (which is not always the case during the tests). OPEAR was tested in a real setting using Contiki emulation. In the tests, as we used an approximation, some performance degradation with respect to the ideal case is measured, and therefore the real lifetime is shorter then the one computed by the offline model. Nevertheless, OPEAR prolongs significantly the network lifetime compared to the benchmark protocol.

We also tested OPEAR on a random graph of 40 nodes, the results obtained in this new set of experiments, leads to similar observations, therefore, due to space limitations we do not include these results.

\section{CONCLUSIONS AND FUTURE WORK}

In this paper we proposed an Optimal Probabilistic Energy Aware protocol for Routing in duty-cycled WSNs (OPEAR). It is designed to be especially useful to improve network lifetime, and therefore reliability, in applications of sensor networks in smart homes for heath care. We compared the performances of our protocol to those of the popular Energy Aware Routing (EAR) protocol by means of emulations of realistic networks, considering different scenarios and different use modes. OPEAR protocol showed to outperform EAR in all settings, allowing to higher network lifetime.

Future research work will cover improved models and algorithms for building accurate probabilistic routing tables, taking into account additional operational details of real networks such as delay and bandwidth limits, as well as providing probabilistic lifetime guarantees. Another extension is a better approximation of contikiMAC, taking also into account the energy of overhearing.

\section{ACKNOWLEDGMENT}

This work was supported in part by the Lorraine region AME Satelor project.

\section{REFERENCES}

[1] H. Cruz-Sanchez, L. Havet, M. Chehaider, and Y.-Q. Song, MPIGate: A Solution to use Heterogeneous Networks for Assisted Living Applications. In 9th IEEE International Conference on Ubiquitous Intelligence and Computing (UIC 2012), pp104-111, Fukuoka, Japan, Sept. 2012.

[2] D. S. J. D. Couto, D. Aguayo, J. Bicket and R. Morris: A HighThroughput Path Metric for Multi-Hop Wireless Routing, MobiCom '03 Proceedings of the 9th annual international conference on Mobile computing and networking, 2013 .

[3] S. Biswas and R. Morris: ExOR: Opportunistic Multi-Hop Routing for Wireless Networks. SIGCOMM '05 Proceedings of the 2005 conference on Applications, technologies, architectures, and protocols for computer communications, 2005 .

[4] V. Angelakis, N. Gazoni and D. Yuan. Probabilistic Routing in Opportunistic Ad Hoc Networks, Wireless Ad-Hoc Networks, Dr. Hongbo Zhou (Ed.), ISBN: 978-953-51-0896-2, InTech, DOI: 10.5772/53997. Available from: http://www.intechopen.com/books/wireless-ad-hocnetworks/probabilistic-routing-in-opportunistic-ad-hoc-networks, 2012.

[5] R. C. Shah, J.M.Rabaey: Energy Aware Routing for Low Energy Ad Hoc Sensor Networks. Wireless Communications and Networking Conference Record, WCNC 2002. 
TABLE III: Simulation details

\begin{tabular}{|c|c|c|c|c|c|c|c|c|c|c|c|c|}
\hline Exper. & \multicolumn{3}{|c|}{$\mathrm{A}$} & \multicolumn{3}{|c|}{$\mathrm{B}$} & \multicolumn{3}{|c|}{$\mathrm{C}$} & \multicolumn{3}{|c|}{$\mathrm{D}$} \\
\hline Cases & (nf) & $(2 \mathrm{f}$ & (4f) & (nf) & $(2 \mathrm{f}$ & $(4 \mathrm{f})$ & (nf) & $(2 \mathrm{f})$ & $(4 f)$ & (nf) & $(2 \mathrm{f})$ & $(4 \mathrm{f})$ \\
\hline Sink & \multicolumn{3}{|c|}{1} & \multicolumn{3}{|c|}{1} & \multicolumn{3}{|c|}{1} & \multicolumn{3}{|c|}{1} \\
\hline Source(s) & \multicolumn{3}{|c|}{20} & \multicolumn{6}{|c|}{$20,19,14$} & \multicolumn{3}{|c|}{ All } \\
\hline Demand & \multicolumn{3}{|c|}{ Uniform } & \multicolumn{3}{|c|}{ Uniform } & \multicolumn{3}{|c|}{ Random } & \multicolumn{3}{|c|}{ Random } \\
\hline Packets/source & \multicolumn{3}{|c|}{$5542-4495$} & \multicolumn{3}{|c|}{$5965-7118$} & \multicolumn{3}{|c|}{$[0.9,1.1] \cdot 7118$} & \multicolumn{3}{|c|}{$[0.9,1.1] \cdot 1915$} \\
\hline Flooding Freq. & - & 2 & 4 & - & 2 & 4 & - & 2 & 4 & - & 2 & 4 \\
\hline Packet Freq. (p/sec) & \multicolumn{3}{|c|}{1.25} & \multicolumn{3}{|c|}{1.25} & \multicolumn{3}{|c|}{1.25} & \multicolumn{3}{|c|}{1.25} \\
\hline Energy Range & \multirow{2}{*}{\multicolumn{3}{|c|}{ [1000-2500] }} & \multicolumn{9}{|c|}{ [2000-3500] } \\
\hline Prob. Distribution & & & & \multicolumn{3}{|c|}{ Uniform } & \multicolumn{3}{|c|}{ Uniform } & \multicolumn{3}{|c|}{ Uniform } \\
\hline
\end{tabular}
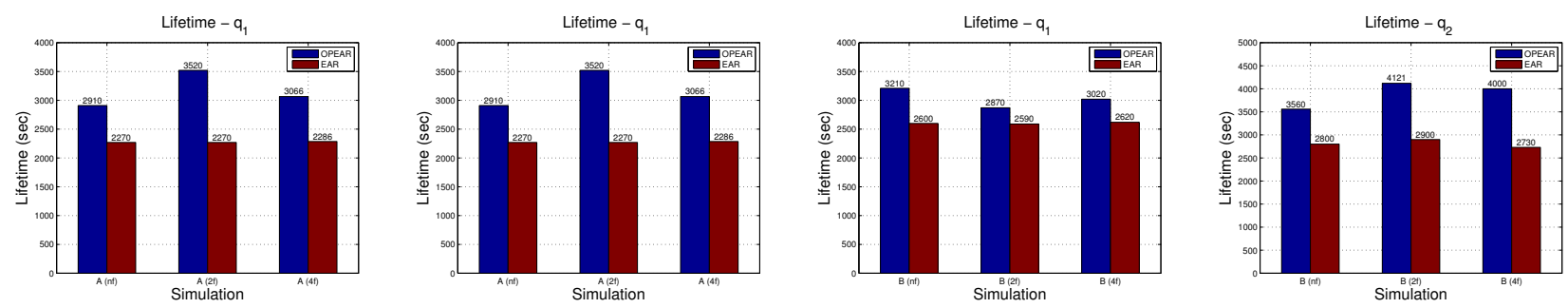

Fig. 3: Network Lifetime a) Exper. A - $q_{i}$, b) Exper. A - $q_{i}^{\prime}$, c) Exper. B - $q_{i}$, d) Exper. B - $q_{i}^{\prime}$
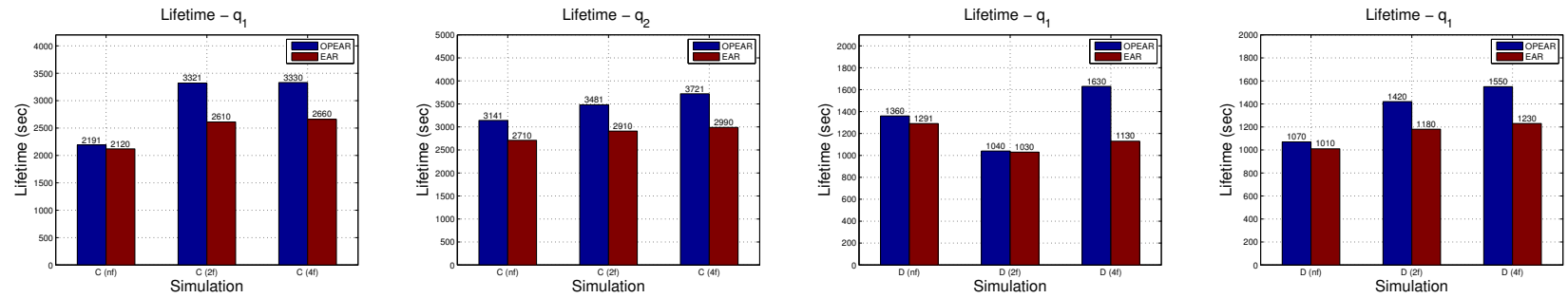

Fig. 4: Network Lifetime a) Exper. C - $q_{i}$, b) Exper. C - $q_{i}^{\prime}$, c) Exper. D - $q_{i}$, d) Exper. D - $q_{i}^{\prime}$

TABLE IV: Variation of Residual Energy

\begin{tabular}{|l|c|c|c|c|c|c|c|c|c|c|c|c|}
\hline Exper. & A (nf) & A (2f) & A (4f) & B (nf) & B (2f) & B (4f) & C (nf) & C (2f) & C (4f) & D (nf) & D (2f) & D (4f) \\
\hline OPEAR & 177456 & 153937 & 132815 & 199666 & 85658 & 128362 & 127919 & 292073 & 165222 & 263149 & 282839 & 251953 \\
\hline EAR & 191083 & 192443 & 191684 & 273326 & 275197 & 303051 & 294316 & 307627 & 329706 & 277622 & 313203 & 318152 \\
\hline \hline
\end{tabular}

[6] A. P. Patil: Design of an energy efficient routing protocol for MANETs based on AODV. International Journal of Computer Science Issues, vol. 8, no. 4, 2011.

[7] P. Nand and S. C. Sharma: Probability based improved broadcasting for AODV routing protocol. In Proceedings of the International Conference on Computational Intelligence and Communication Systems (CICN'11), pp. 621-625, October 2011.

[8] Sang-Hyun Park, Seungryong Cho, and Jung-Ryun Lee: Energy-Efficient Probabilistic Routing Algorithm for Internet of Things. Journal of Applied Mathematics, vol. 2014, Article ID 213106, 7 pages, 2014.

[9] M. Bhaedwaj, A. P. Chandrakasan : Bounding the Lifetime of Sensor Networks Via Optimal Role Assignments, INFOCOM 2002.

[10] H. U. Yildiz, M. Temiz and B. Tavli: Impact of Limiting Hop Count on the Lifetime of Wireless Sensor Networks, Signal Processing and Communications Applications Conference (SIU), 2013.

[11] C. Schurgers and M. Srivastara: Energy efficient routing in wireless sensor networks, MILCOM Proceedings on Communications for NetworkCentric Operations: Creating the Information Force, 2001.

[12] F. Osterlind, A. Dunkels, J. Eriksson, N. Finne, and T. Voigt. CrossLevel Sensor Network Simulation with COOJA. In $31^{\text {st }}$ IEEE Conference on Local Computer Networks (LCN), pages 641-648, Tampa, FL, USA, November 2006
[13] A. Brandt, J. Buron, and G. Porcu, "Home Automation Routing Requirements in Low-Power and Lossy Networks", IETF, RFC 5826,2010

[14] V. Chvátal: Linear Programming, W. H. Freeman, 1983.

[15] R.K. Ahuja, T.L. Magnanti, and J.B. Orlin: Network Flows: Theory, Algorithms, and Applications, Prentice Hall, 1993.

[16] Adam Dunkels. The ContikiMAC Radio Duty Cycling Protocol. Technical Report T2011:13, Swedish Institute of Computer Science, December 2011.

[17] Multi Protocol Interface Gateway website, http://mpigate.loria.fr/, last access Oct. 23rd, 2015.

[18] W. Ye, J. Heidemann, D. Estin: Medium access control with coordinated adaptive sleeping for wireless sensor networks, IEEE/ACM Transactions on Networking (TON), Volume 12 Isuue 3, June 2004.

[19] Q. Wang, W. Yang: Energy Consumption Model for Power Management in Wireless Sensor Networks, SECON 2007: 142-151

[20] S. Prayati, D. Antonopoulos, T. Stoyanova, C. Koulamas, G. D. Papadopoulos: A modeling approach on the TelosB WSN platform power consumption. Journal of Systems and Software 83(8): 1355-1363 (2010)

[21] http://www.ti.com/lit/ds/symlink/cc2420.pdf

[22] Contiki project website, http://www.contiki-os.org, last access Oct. 23rd, 2015 